\title{
Sustained delivery of nicotinamide limits cortical injury and improves functional recovery following traumatic brain injury
}

\author{
Andrea M. Goffus, ' Gail D. Anderson² and Michael R. Hoane ${ }^{1, *}$ \\ 'Restorative Neuroscience Laboratory; Center for Integrative Research in Cognitive and Neural Sciences; Department of Psychology; Southern Illinois University; Carbondale, \\ IL USA; ${ }^{2}$ Department of Pharmacy; University of Washington; Seattle, WA USA
}

Key words: neurorehabilitation, therapy, brain injury, behavior, restorative neurology

\begin{abstract}
Abbreviations: TBI, traumatic brain injury; CCI, cortical contusion injury; NAM, nicotinamide; MWM, morris water maze; PARP, poly-ADP-ribose polymerase; $\mathrm{NAD}^{+}$, nicotinamide adenine dinucleotide; $\mathrm{BBB}$, blood-brain barrier; $\mathrm{ATP}$, adenosine triphosphate; FPI, fluid percussion injury; ANOVA, analysis of variance; ad lib, ad libitum
\end{abstract}

\begin{abstract}
Previously, we have demonstrated that nicotinamide (NAM), a neuroprotective soluble B-group vitamin, improves recovery of function following traumatic brain injury (TBI). However, no prior studies have examined whether NAM is beneficial following continuous infusions over 7 days post-TBI. The purpose of this study was to investigate the preclinical efficacy of NAM treatment as it might be delivered clinically; over several days by slow infusion. Rats were prepared with either unilateral controlled cortical impact (CCI) injuries or sham procedures and divided into three groups: $\mathrm{CCl}-\mathrm{NAM}, \mathrm{CCl}-$ vehicle and sham. Thirty minutes following $\mathrm{CCl}$, Alzet osmotic mini-pumps were implanted subcutaneously. NAM was delivered at a rate of $50 \mathrm{mg} / \mathrm{kg} /$ day for 7 days immediately post-CCl. On day 7 following injury, the pumps were removed and blood draws were collected for serum NAM and nicotinamide adenine dinucleotide (NAD ${ }^{+}$analyses. Starting on day 2 post-CCl, animals were tested on a battery of sensorimotor tests (bilateral tactile adhesive removal, locomotor placing and limb-use asymmetry). Continuous infusion of NAM resulted in a significant serum elevation in NAM, but not $\mathrm{NAD}^{+}$. Statistical analyses of the tactile removal and locomotor placing data revealed that continuous administration of NAM significantly reduced the initial magnitude of the injury deficit and improved overall recovery compared to the vehicle-treated animals. NAM treatment also significantly decreased limb-use asymmetries compared to vehicle-treated animals. The overall extent of the cortical damage was also reduced by NAM treatment. No detrimental effects were seen following continuous infusion. The present results suggest that NAM delivered via a clinically relevant therapeutic regimen may truncate behavioral damage following TBI. Thus our results offer strong support for translation into the clinical population.
\end{abstract}

\section{Introduction}

Traumatic brain injury (TBI) is a major public health crisis. An estimated 1.4 million people incur a TBI annually; a rate of 1 injury every 23 seconds. ${ }^{1}$ The resulting incidence rate is approximately 100 per 100,000 US citizens and is respectively 8 and 34 times higher than breast cancer and HIV/AIDS diagnoses each year. ${ }^{2}$ Military personnel, particularly those serving in Afghanistan and Iraq are a special population highly susceptible to TBI as blasts are the primary cause of injury for this group. ${ }^{3}$ Sports related head injuries are also becoming more common and it is estimated that 300,000 such injuries occur annually. ${ }^{1}$ Despite these staggering statistics, no clinically effective pharmacological treatments exist to aid recovery during the acute or secondary stages of injury.-6

A prevalent issue in the field of TBI research is the number of therapeutic compounds that have initially proven experimentally promising, but then do not translate clinically. It has been stated that there is a gap between the bench to the bedside. ${ }^{5}$ In other words, inconsistencies exist between preclinical testing in the laboratory and the clinic. The gaps most relevant to experimentalists are: treatment window and duration. ${ }^{4}$ For animal models the treatment window (in which the pharmacotherapy begins) is approximately less than an hour while clinically it is usually four to eight hours; while treatment durations can be over several days. Surprisingly few studies have examined continuous dosing regimens in TBI. Subcutaneous osmotic mini-pumps were used in order to deliver the immunosuppressant cyclosporine A (CsA) following cortical contusion injury (CCI). ${ }^{7}$ In a second study, a single bolus intraperitoneal (i.p.) injection, as a loading dose, was administered 15 mins post-CCI followed by chronic infusion of CsA. In another study, nicotine was infused continuously following TBI and it was shown to attenuate nicotinic receptor deficits 


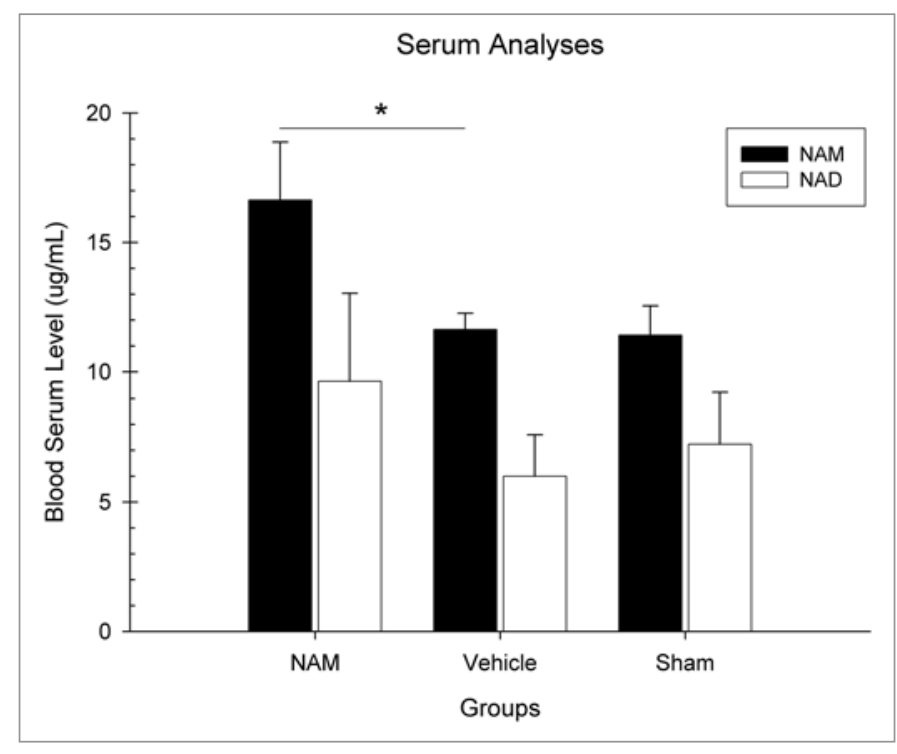

Figure 1. Continuous infusion of nicotinamide (NAM) significantly elevated serum NAM but not NAD+ levels following cortical contusion injury (CCI). Plotted are the mean (+SEM) blood serum levels $(\mu \mathrm{g} / \mathrm{mL})$ of NAM and NAD ${ }^{+}$determined by HPLC for the NAM-treated, vehicle-treated, and sham animals collected following 7 days of continuous infusion of NAM ( $50 \mathrm{mg} / \mathrm{kg} /$ day, s.c. minipump). NAM was significantly elevated $\left({ }^{*} \mathrm{p}<0.05\right)$ compared to vehicle; whereas, $N A D^{+}$levels were only slightly elevated.

following injury. ${ }^{8}$ In addition, continuous delivery of progesterone by silastic capsules following TBI has been shown to reduce anxiety. ${ }^{9}$ All of these studies demonstrated significant treatment effects; however, few studied the effect on recovery of function following injury. These studies used subcutaneous infusions as a clinically relevant route of administration since continuous intravenous infusion in rodents is problematic. Therefore, these studies could not conclude whether continuous infusions result in prolonged and enhanced recovery of function following TBI.

A promising preclinical treatment for TBI is nicotinamide (NAM), a neuroprotective soluble B-group vitamin. ${ }^{10-17}$ The administration of an i.p. dose of NAM $(500 \mathrm{mg} / \mathrm{kg})$ administered 15 minutes and 24 hours post-TBI, resulted in significant behavioral and anatomical recovery following bilateral frontal injury. ${ }^{10}$ Using the same dosing regimen, NAM was able to prevent bloodbrain barrier (BBB) breakdown and reduce apoptosis at 5, 24 and 72 hours post injury following unilateral CCI. ${ }^{12}$ In a model of fluid percussion injury (FPI), 2 doses of 50 or $500 \mathrm{mg} / \mathrm{kg}$ NAM administered within 24 hrs of injury, induced sensorimotor and cognitive recovery up to thirty-five days post injury ${ }^{15}$ and provided significant acute neuroprotection in the injured cortex 24 hours post-FPI. ${ }^{11}$ The window of opportunity for NAM treatment $(50 \mathrm{mg} / \mathrm{kg}$, i.p.) has been shown to extend to $4-6$ hours post-TBI in the bilateral frontal CCI model on both sensorimotor and cognitive measures and in some cases can be effective up to $24 \mathrm{hrs}$ post-TBI on sensorimotor behaviors in a unilateral CCI model. ${ }^{13-14}$ However, no previous studies have examined whether NAM is beneficial following continuous administration. Future clinical studies with NAM will likely utilize continuous infusions and thus need to be examined preclinically. Since continuous i.v. infusion into awake, behaving rodents is problematic at best, the alternative is the use of subcutaneous osmotic mini-pumps. Therefore, the main purpose of this study was to investigate the preclinical efficacy of NAM treatment at a targeted delivery rate of $0.625 \mathrm{mg} / \mathrm{hr}$ (to achieve $50 \mathrm{mg} / \mathrm{kg}$ ), as it would be delivered clinically; over several days by slow, continuous infusion.

\section{Results}

NAM and NAD ${ }^{+}$analysis. Two separate one-way ANOVAs were conducted to analyze NAM and $\mathrm{NAD}^{+}$blood serum levels $(\mu \mathrm{g} / \mathrm{mL})$. Three levels were used in this analysis (NAM-treated, vehicle-treated and sham). There was a statistically significant difference between the three groups in terms of NAM blood serum levels, $F(2,20)=3.81, p=0.04$ (see Fig. 1). Pairwise comparisons revealed significant differences in serum NAM levels $[\operatorname{LSD}(15)=5.00, \mathrm{p}=0.02]$ between the NAM $(\mathrm{M}=$ 16.63 , $\mathrm{SEM}=2.24)$ and vehicle-treated groups $(\mathrm{M}=11.62$, SEM $=0.62$ ). Therefore, injured animals treated with chronic infusions of NAM had higher serum NAM levels than the injured animals treated with vehicle. The NAD blood serum level analysis revealed no overall significant differences between groups $\mathrm{F}(2,20)=0.46, \mathrm{p}=0.64$; however, there was a differences between the NAM $(\mathrm{M}=9.63, \mathrm{SEM}=3.82)$ and vehicletreated groups $(\mathrm{M}=6.10, \mathrm{SEM}=1.60$ (see Fig. 1 ).

Bilateral tactile removal. Latency to remove the tactile stimuli (adhesive patches) was analyzed in a $3 \times 6$ mixed ANOVA. The factors were group (NAM-treated, vehicle-treated and sham) $\mathrm{x}$ day (1-6). Latency to remove the contralateral patch was not significantly affected by days $\mathrm{F}(4.44,88.73)=1.61, \mathrm{p}=0.17$. The group by day interaction was also not statistically significant $F(8.87,88.73)=0.96, p=0.48$. However, the main effect of group was statistically significant, $F(2,20)=4.55, p=0.02$. The chronic infusion of NAM significantly reduced the removal latencies in lesioned animals (see Fig. 2). Fischer's LSD pairwise comparisons revealed significant differences in removal latencies between the NAM-treated group and the vehicle-treated group on days $2(\operatorname{LSD}(15)=44.50, \mathrm{p}=0.006), 4(\operatorname{LSD}(15)=32.63$, $\mathrm{p}=0.04)$ and $6(\operatorname{LSD}(15)=36.44, \mathrm{p}=0.01)$. No significant injury-induced impairments in patch removal were seen on the left forelimb $\mathrm{p}>0.05$.

Locomotor placing. Foot-faults on the grid floor were analyzed in a $3 \times 6$ mixed ANOVA. The factors were group (NAMtreated, vehicle-treated and sham) $\mathrm{x}$ day (1-6). The number of foot-faults was significantly affected by days $\mathrm{F}(3.76,75.11)$ $=3.22, \mathrm{p}=0.001$ and group, $\mathrm{F}(2,20)=63.17, \mathrm{p}=0.001$ (see Fig. 3). However, the interaction was not statistically significant $\mathrm{F}(7.51,75.11)=0.57, \mathrm{p}=0.79$. Pairwise comparisons revealed significant differences in foot-faults between the NAM-treated group and the vehicle-treated group on days $2(\operatorname{LSD}(15)=24.63$, $\mathrm{p}=0.008), 4(\operatorname{LSD}(15)=35.04, \mathrm{p}=0.001), 6(\operatorname{LSD}(15)=28.25$, $\mathrm{p}=0.005), 8(\operatorname{LSD}(15)=22.91, \mathrm{p}=0.001), 14(\operatorname{LSD}(15)=16.16$, $\mathrm{p}=0.01)$ and $28(\operatorname{LSD}(15)=31.20, \mathrm{p}=0.001)$.

Limb-use asymmetry. The percentage of bias towards use of the contralateral forelimb was analyzed in a $3 \times 3$ mixed 
ANOVA. The factors were group (NAM-treated, vehicle-treated and sham) $\mathrm{x}$ day (1-3). The percentage of contralateral forelimb bias was not statistically significantly affected by day, $F(2,40)=$ $0.75, p=0.48$. The interaction between day and group was also not statistically significant, $F(4,20)=1.04, p=0.40$. However, the group factor was statistically significant $F(2,20)=32.90$, $p$ $=0.001$ (see Fig. 4). Pairwise comparisons revealed significant differences in contralateral biases between the NAM-treated and vehicle-treated groups on day $4[\operatorname{LSD}(15)=11.32, \mathrm{p}=0.04]$, $15[\operatorname{LSD}(15)=15.78, \mathrm{p}=0.007]$ and $28[\operatorname{LSD}(15)=11.33, \mathrm{p}=$ 0.05]. Therefore, chronic administration of NAM significantly reduced the bias towards the contralateral forelimb.

Lesion analysis. An initial one-way ANOVA was performed on the volume of the intact contralateral cortex. Three groups were used in this analysis (NAM-treated, vehicle-treated and sham). There was not a significant difference in contralateral cortical volume between the groups, $\mathrm{F}(2,20)=2.36, \mathrm{p}=0.12$. A second, one-way ANOVA was used to analyze the percentage of cortex volume reduction in the contralateral hemisphere compared to the ipsilateral hemisphere. There was a significant lesion effect between the groups, $\mathrm{F}(2,20)=80.84, \mathrm{p}=0.001$ (see Fig. 5). Pairwise comparisons revealed significant differences in cortex reduction $(\operatorname{LSD}(15)=6.38, \mathrm{p}=0.01)$ between the NAM $(\mathrm{M}=$ $21.91, \mathrm{SEM}=1.45)$ and vehicle-treated groups $(\mathrm{M}=28.29, \mathrm{SEM}$ $=2.33$ ). Therefore, lesioned animals treated with chronic infusions of NAM had significantly lower percentage of reduction in the contralateral cortex than the lesioned animals treated with vehicle. Representative histology sections are shown in Figure 6.

\section{Discussion}

The purpose of this study was to examine the preclinical efficacy of continuous treatment with NAM following TBI. The preclinical efficacy of NAM following TBI has been well established for bolus administrations; however, it is of clinical interest to determine if sustained, continuous infusion of NAM also demonstrates preclinical efficacy.

The sensorimotor testing on the bilateral tactile adhesive removal test following unilateral CCI of the SMC resulted in strong initial injury impairments in the vehicle-treated animals. In contrast, continuous infusion of NAM resulted in a significant reduction in the initial magnitude of the behavioral deficit and facilitation in recovery of function. On the locomotor placing test, unilateral CCI produced enduring deficits in the vehicle-treated animals. Infusion of NAM significantly reduced the magnitude of the initial placing deficit; in fact, NAM-treated animals were significantly less impaired on every test day compared to the vehicle-treated animals. On the forelimb asymmetry test CCI produced very enduring deficits in the vehicle-treated animals. On all test days (4, 15 and 28 days) post-injury the vehicle-treated animals showed a forelimb bias favoring the unimpaired forelimb that was greater than $80 \%$. In contrast, the sham controls showed virtually no bias and used both the ipsilateral and contralateral forelimbs equally. The animals treated with infusion of NAM demonstrated a significant reduction in their bias toward the unimpaired limb on each test day compared to
Tactile Removal Test

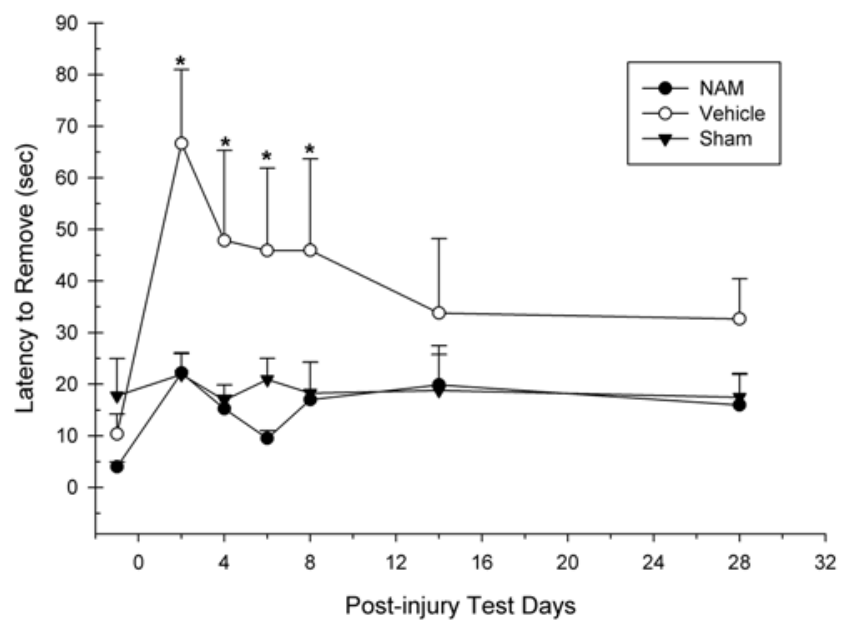

Figure 2. Continuous infusion of nicotinamide (NAM) significantly improved recovery on the bilateral adhesive removal test following cortical contusion injury (CCI). The graph shows the plotted mean (+SEM) of the tactile stimuli removal latencies for all three experimental groups (NAM-treated, vehicle-treated and sham) following unilateral CCI. Seven days of continuous infusion of NAM significantly improved performance compared to vehicle $\left({ }^{*} p<0.05\right)$. There were no statistically significant differences between the NAM-treated and sham animals.

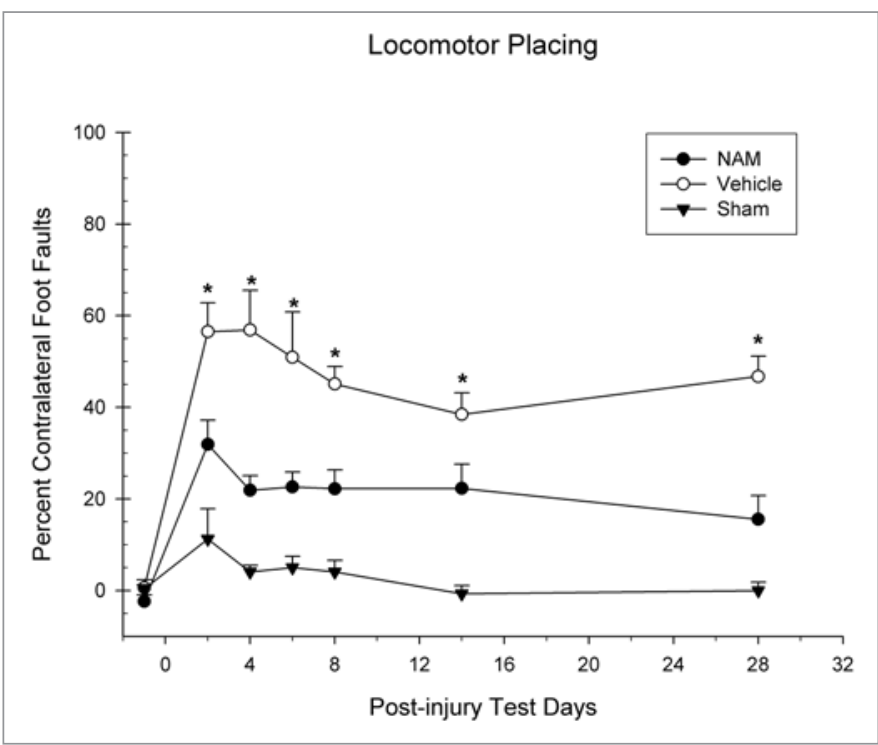

Figure 3. Continuous infusion of nicotinamide (NAM) significantly improved recovery on the locomotor placing test following cortical contusion injury (CCI). The graph shows the plotted mean (+SEM) of the percentage of foot-faults on the grid for all three experimental groups (NAM-treated, vehicle-treated, and sham) following unilateral CCI. Seven days of continuous infusion of NAM significantly improved locomotor placing performance compared to vehicle $\left({ }^{*} p<0.05\right)$. Although, a significant treatment effect was seen with NAM compared to vehicle in the injured animals. 


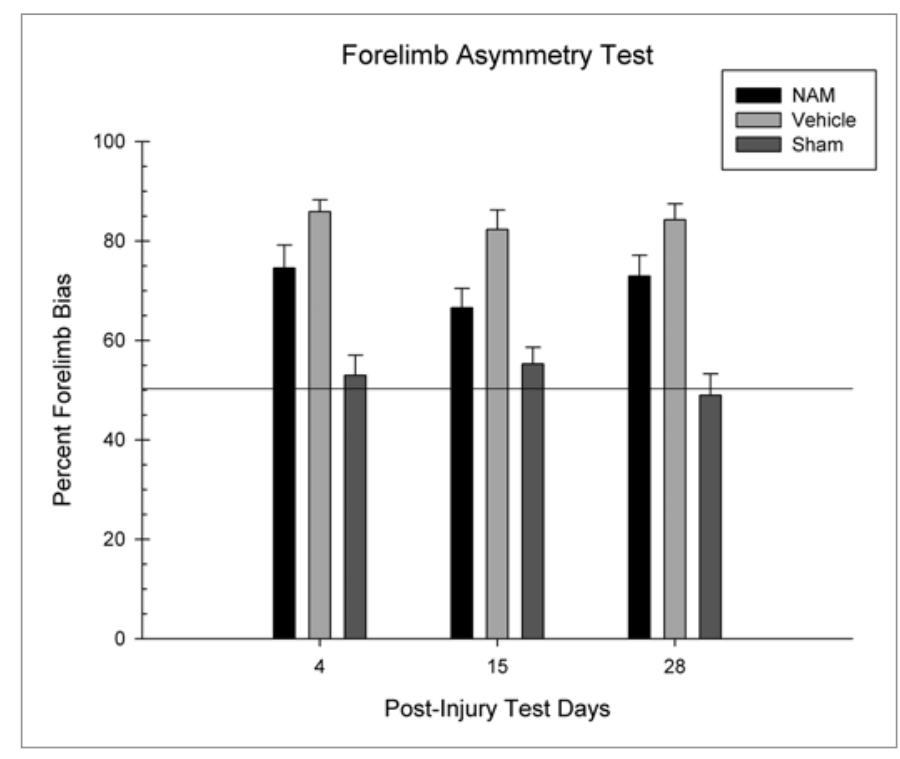

Figure 4. Continuous infusion of nicotinamide (NAM) significantly improved recovery on the forelimb asymmetry test following cortical contusion injury (CCI). The graph shows the plotted mean (+SEM) of the percent forelimb bias for all three experimental groups (NAM-treated, vehicle-treated and sham) following unilateral $\mathrm{CCl}$. The no bias line indicates the point at which the ipsilateral and contralateral forelimbs are being used equally. Seven days of continuous infusion of NAM significantly improved performance compared to vehicle. Although, a significant treatment effect was seen with NAM compared to vehicle in the injured animals, there were still enduring deficits compared to the sham controls. the vehicle-treated animals. Together, all of the behavioral data in this study suggest that a 7-day regimen of low dose NAM significantly improved recovery of function following TBI.

The behavioral data as a whole in this study replicate and extended the beneficial effects we have seen with bolus administrations of NAM following CCI and fluid percussion injury (FPI) on a multitude of behavioral measures, including motor, sensorimotor and cognitive tests. ${ }^{10,13-15}$ In the present study direct comparison between bolus administrations of NAM and continuous administration of NAM were not performed, so experimentally, it cannot be determined if the continuous infusions were better than the bolus route. However, some speculations can be made. The current continuous infusion findings are consistent with a previous study in which bolus administrations of NAM $(50 \mathrm{mg} / \mathrm{kg})$ were performed daily for 5 days post-CCI. ${ }^{13} \mathrm{On}$ the bilateral tactile removal test, animals receiving injections at 15 minutes or 4 and 8 hours post-CCI showed recovery over time, with the best recovery shown in the 15 minute group. However, in the current study, the NAm-treated group demonstrated a greater degree of initial injury reduction on day 2 post-CCI. A strong initial reduction in magnitude of the tactile removal deficit has also been shown following 12 days of bolus injections of $50 \mathrm{mg} / \mathrm{kg}$ NAM. ${ }^{14}$ However, in that paper animals did not start to perform at sham control levels until day 14 post-CCI; whereas, in the current study NAM-treated animals were performing at sham levels on day 2. A similar effect was also seen on the locomotor placing test. Bolus injections of NAM administered for 12 day starting 4 hours post-CCI resulted in the animals performing at sham control levels. ${ }^{14}$ When treatment was initiated 8 and 24 hours after injury, the animals did not perform at sham levels, but were lower than vehicle-treated animals. ${ }^{14}$ In the present study, animals treated with NAM favored the use of their contralateral forelimb significantly less than vehicle-treated animals. A similar finding showing that NAM treatment when started at 4 or 8 hours post-CCI demonstrated a significant reduction in contralateral forelimb bias has been reported. ${ }^{14}$ Thus, the results from the current study continue to support the preclinical efficacy of NAM for TBI. However, in our experience the beneficial behavioral effects we saw in the current study are some of the strongest we have observed in these animals models.

The data from the lesion analysis also revealed a significant improvement in tissue integrity. Infusion of NAM significantly reduced the size of the injury, as measured by a cortical volume analysis. The vehicle-treated group had an average reduction in frontal cortical volume of $28.3 \%$ compared to the NAM-treated group that showed a reduction of $21.9 \%$. This cortical protective effect is similar to those effects we have seen in previous studies. ${ }^{10,11,13-16}$ Furthermore, previous studies in our laboratory have shown NAM to significantly reduce apoptosis, GFAP expression, edema and neuronal death. ${ }^{11,12,16}$ Thus, it is likely that by reducing the pathophysiological events associated with CCI in previous studies that NAM resulted in the beneficial behavioral effects observed in the current study.

We have previously demonstrated that systemic administration of NAM elevated serum NAM concentrations. ${ }^{13,15}$ The data from the current study confirmed that sustained, low-level delivery of
Figure 5. Continuous infusion of nicotinamide (NAM) significantly limited cortical injury following cortical contusion injury (CCI). Plotted are the mean (+SEM) percentages of cortical volume reductions in the NAM-treated animals, vehicle-treated animals, and sham animals. NAM significantly reduced the extent of the cortical injury compared to vehicle $\left({ }^{*} \mathrm{p}<0.05\right)$. 


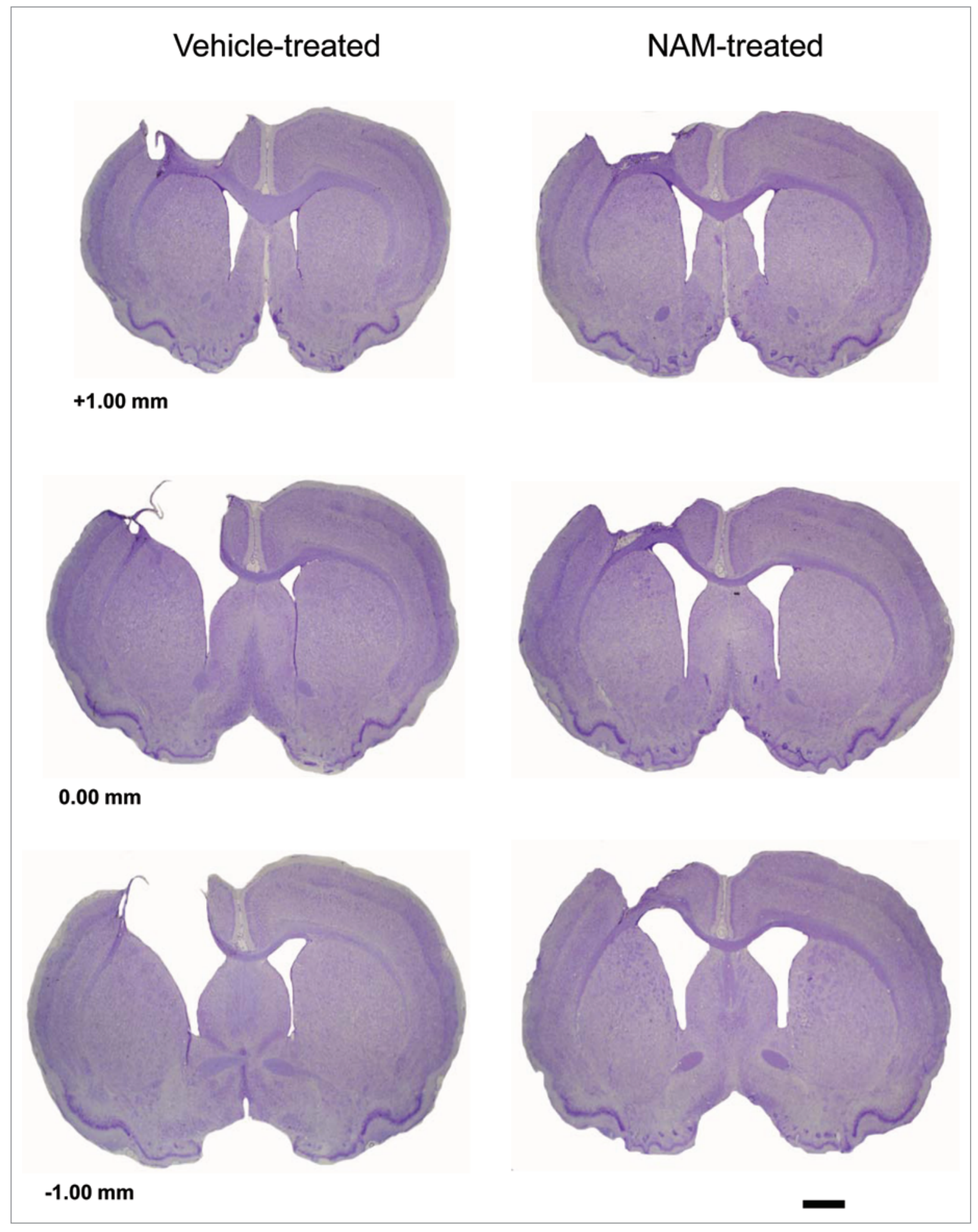

Figure 6. Representative histology showing that continuous infusion of nicotinamide (NAM) significantly improved recovery on the forelimb asymmetry test following cortical contusion injury (CCI). Unilateral CCI produced marked cortical cavitation. Shown are representative images of the effect of NAM infusion and vehicle treatment through the extent of the injury $(0.44 \mathrm{x})$ at coordinates $+1.00,0.00$ and $-1.00 \mathrm{~mm}$ relative to bregma. Measurement bar $=2.0 \mathrm{~mm}$.

NAM for 7 days also increased serum NAM levels significantly. Serum $\mathrm{NAD}^{+}$levels were also analyzed and no such significant elevation was seen in the blood following NAM administration.
Interestingly, there were lower levels of $\mathrm{NAD}^{+}$at 7 days following injury in the vehicle-treated animals compared to the sham controls; furthermore, treatment with NAM appeared to have 
partially offset this reduction. One factor that may have limited our ability to see significant changes in serum $\mathrm{NAD}^{+}$levels is the fact that blood was collected 7 days post-TBI, it might be expected that larger injury-induced decreases in serum $\mathrm{NAD}^{+}$ could be observed in the acute, early phases after injury. A recent article has shown that administration of a poly(ADP-ribose) polymerase inhibitor (PARP) prevented $\mathrm{NAD}^{+}$depletion in the injured cortex acutely following TBI. ${ }^{18}$ Thus, it is likely that a time course analysis of the tissue might reveal different results from those gathered with the serum analysis and it is not known how cortical levels of $\mathrm{NAD}^{+}$correlate with serum levels. A study investigating this relationship is currently being conducted.

NAM has been shown to be a broad spectrum neuroprotectant, which is mechanistically multi-dimensional, and has expanding avenues into a variety of health conditions. ${ }^{19-21}$ NAM has shown promise experimentally as a poly (ADP-ribose) polymerase (PARP) inhibiter ${ }^{21-23}$ and has shown increases in cell survival, adenosine triphosphate (ATP) and $\mathrm{NAD}^{+}$levels as well. ${ }^{24-26}$ NAM also has a role as an essential precursor for the coenzyme NAD ${ }^{+}{ }^{27} \mathrm{NAM}$ is able to maintain levels of PARP therefore supplying neuronal preservation and energy. NAM is able to reach peak levels within the brain fairly quickly and therefore readily increase $\mathrm{NAD}^{+}$and ATP brain stores via a specific uptake mechanism. ${ }^{28}$ NAM can be used to alleviate two detrimental cascades after injury: elevate $\mathrm{NAD}^{+}$thereby restoring ATP levels and to serve as a PARP inhibitor, also restoring ATP. ${ }^{22}$ Using NAM to elevate $\mathrm{NAD}^{+}$levels in conjunction with PARP inhibition are additive in respect to ATP restoration and has proven to be more beneficial. ${ }^{29}$ Additionally, NAM is critical for synthesizing nicotinamide adenine dinucleotide phosphate $\left(\mathrm{NADP}^{+}\right)$, modulating cellular metabolism, plasticity, longevity, inflammatory microglial responses, and maintenance of the internal and external cellular environment. ${ }^{27,30}$ Recently, new cellular pathways have emerged that appear to govern the beneficial actions of NAM in disease conditions and have recently been reviewed. ${ }^{20}$ These pathways include forkhead transcription factors and the sirtuins. ${ }^{19,20}$ NAM also activates protein kinase B and inhibits transcription of proapoptotic genes. A downstream effect of protein kinase B is closing of the mitochondrial transition pores opened by excessive $\mathrm{Ca}^{2+}$ influx. The mitochondrion is able to regain ionic homeostasis and bring cytochrome $C$ back to physiological conditions thereby inhibiting caspase 9 mediated cell death..$^{20,27}$

In our experience with NAM therapy for experimental TBI we have not observed any detrimental effects of treatment at 50 or $500 \mathrm{mg} / \mathrm{kg}$. ${ }^{10-17}$ These studies have included daily dosing $\mathrm{r}$ egimens of NAM $(50 \mathrm{mg} / \mathrm{kg})$ for 7 and 14 days. ${ }^{13,14}$ In the current study, continuous infusion of NAM did not result in any overtly observable detrimental effects. Currently, NAM is used clinically in the treatment of pellagra (nicotinamide deficiency) and diabetes. Doses less than $600 \mathrm{mg} / \mathrm{kg}$ have been used daily for up to several weeks in studies using rats without complications, but the at higher doses liver and kidney damage have been reported. ${ }^{26}$ For treatment of various diseases in the human population, daily doses of 1 to 12 grams have been used. ${ }^{31}$ Drug safety tests indicate that daily doses up to 3 grams can be used long-term safely and without side effects. ${ }^{31}$ However, a recent reports suggests that there is some concern that NAM therapy may be a double-edged sword when treating oxidative or metabolic conditions, especially in the aged populations. ${ }^{19,20}$ Current studies in our laboratory are examining the ability of NAM to be neuroprotective in aged animals.

In conclusion, the current study showed that unilateral SMC injury produced relatively enduring behavioral deficits on a variety of forelimb sensorimotor tasks. Sustained delivery of NAM throughout the first 7 days post-injury resulted in significant improvements in these behavioral deficits. Future studies will be aimed at maximizing NAM administration by providing sustained, steady-state levels of treatment with NAM post-TBI.

\section{Methods}

Animals. Twenty-three male Sprague-Dawley rats were used for this study and weighed an average of $369.60 \mathrm{~g}$ (SEM 7.42) at the time of injury and pump implantation. A single animal in the sham group died during recovery and was not replaced. The experimental procedures conducted within this study were reviewed and approved by the Institutional Animal Care and Use Committee and the study was conducted in a facility certified by the American Association for the Accreditation of Laboratory Animal Care. Animals were individually housed in standard cages and allowed ad lib access to food and water. A twelve hour light:dark cycle was used. Rats were handled daily for approximately one week before surgery. Behavioral testing took place during the light cycle.

CCI surgery. Surgical procedures were based on previous studies ${ }^{13,14}$ and performed under aseptic conditions. Animals were anesthetized with a combination of Isofluorane (2-4\%) and oxygen $(0.8 \mathrm{~L} /$ minute $)$ and placed on a heated surgical stage $\left(37^{\circ} \mathrm{C}\right)$ in a stereotaxic frame. A unilateral CCI over the left sensorimotor cortex or sham procedure was then performed. A midline incision was made through the skin and underlying fascia. A craniotomy measuring $5.0 \mathrm{~mm}$ in diameter was created using a motor tool. A specially designed drill bit was used to prevent damage to the meninges and cortex. The craniotomy was positioned at Ap $=0.0, \mathrm{ML}=+2.5$. The cortical region containing the sensorimotor cortex (forelimb region) was then exposed. A sterile, stainless steel impactor tip with a $4.0 \mathrm{~mm}$ diameter was used to create the injury. The tip was attached to an electromagnetic impactor (www.myneurolab.com) and situated at a 10 degree angle. The tip made impact with the cortex at a velocity of $2.75 \mathrm{~m} / \mathrm{s}$ for 0.5 seconds. A $2.0 \mathrm{~mm}$ compression of the cortex occurred. After injury, bleeding was controlled and incisions were sutured closed. Afterwards, animals were placed in a heated recovery cage until locomotion returned. Sham procedures were the same, except cortical impact did not occur.

Drug administration. Twelve hours prior to surgery, Alzet osmotic mini-pumps (model 2001; delivery rate of $1 \mu \mathrm{L}$ per hour for 7 days) were primed and loaded with a concentration of NAM $(625 \mathrm{mg}$ of NAM dissolved per $1 \mathrm{~mL}$ of $0.9 \%$ PBS) or $0.9 \%$ PBS. The pumps were then incubated overnight. Approximately thirty minutes after CCI, the osmotic mini- 
pumps were implanted (subcutaneous, s.c.). Briefly, under gas anesthesia an incision was made just posterior to the shoulder blades. After the incision was made, the underlying fascia and connective tissue was separated from the skin and the osmotic mini-pump was placed within the "pocket." Afterwards, the incision was sutured closed. The osmotic mini-pumps delivered the NAM or vehicle at a rate of $50 \mathrm{mg} / \mathrm{kg}$ per day for seven days following CCI, based on the weight of the animal at the time of injury. On day seven the animals were again anesthetized and the pumps were removed and blood was collected from the tail vein for NAM and NAD ${ }^{+}$serum analysis. There were three groups. Group 1 received CCI and was treated with an osmotic mini-pump containing NAM (NAMtreated; $\mathrm{n}=8)$. Group 2 also received CCI and was treated with an osmotic mini-pump containing the vehicle solution $(0.9 \%$ PBS; vehicle-treated; $\mathrm{n}=8$ ). Group 3 received sham procedures and an osmotic mini-pump containing vehicle solution (sham; $\mathrm{n}=7$ ). See Table 1 .

Bilateral tactile removal. In order to test somatosensory function this test was administered on pre-injury day 2 and post-CCI days 2, 4, 6, 8, 14 and $28 .{ }^{13,14}$ Small adhesive patches (approximately $113.0 \mathrm{~mm}^{2}$ ) were applied to the radial aspects of each forelimb. The animal was then returned to their home cage. The latency for removal of the patch was recorded for each forelimb and was recorded with a stopwatch. A trial ended after both patches were completely removed or 120 seconds elapsed. Animals received two trials per test day. The latency to remove the adhesive patch from the contralateral forelimb was the dependent variable of interest.

Locomotor placing. In order to assess recovery of coordinated locomotor forelimb movement, this test was administered on preinjury day 2 and post-CCI days 2, 4, 6, 8, 14 and 28. ${ }^{14,15}$ On each test day, rats were placed on an elevated grid floor $(56.0 \times 54.0$ $\mathrm{cm})$ and allowed to explore for three minutes. There were openings measuring $3.5 \mathrm{~cm}^{2}$ in size on the grid floor. A "foot-fault" occurred when a rat inaccurately placed the forelimb through these openings. Rats were administered one trial per test day. The number of contralateral and ipsilateral forelimb "foot-faults" was the dependent variable. The following equation was used to calculate the percentage of contralateral foot-faults: $\{[$ (Contralateral foot-faults-ipsilateral foot-faults)/number of steps during the trial $\left.]^{*} 100\right\}$.

Limb-use asymmetry. To assess recovery of impaired (contralateral) forelimb use, this test was administered on post-CCI days 4, 15 and 20. ${ }^{14,32}$ Rats were placed in a clear observation chamber $(20 \mathrm{~cm} \times 20 \mathrm{~cm} \times 30 \mathrm{~cm})$ with four vertical surfaces. Animals were given a single five minute trial on each test day and allowed to place their paws on the vertical surface. The number of ipsilateral and contralateral paw placements on the vertical surface was the dependent variable. The following equation was used to calculate the percent forelimb bias: $\{[$ ipsilateral paw placements + (both paw placements at once/2)]/[ipsilateral + contralateral + both paw placements] $\left.{ }^{*} 100\right\} .{ }^{33}$

NAM and NAD ${ }^{+}$analysis. These methods were based on previous studies. ${ }^{22,23}$ Immediately following removal of the pumps, blood samples were collected from the animals' tail vein. The
Table 1. Experimental groups and timeline

\begin{tabular}{|c|c|c|}
\hline Groups & Condition & n's \\
\hline Group 1 & $\mathrm{CCl}+\mathrm{NAM}$ & 8 \\
\hline Group 2 & $\mathrm{CCl}+$ Vehicle & 8 \\
\hline Group 3 & Sham + Vehicle & 7 \\
\hline \multicolumn{3}{|c|}{ Timeline } \\
\hline \multicolumn{2}{|c|}{ Handling and pre-testing } & Days -7 to 0 \\
\hline \multicolumn{2}{|c|}{ Surgery } & Day 0 \\
\hline \multicolumn{2}{|c|}{ Pump Implantation } & 30 minutes \\
\hline \multicolumn{2}{|c|}{ Bilateral adhesive removal test } & Days $2,4,6,8,14,28$ \\
\hline \multicolumn{2}{|c|}{ Locomotor placing test } & Days $2,4,6,8,14,2 \varepsilon$ \\
\hline \multicolumn{2}{|c|}{ Forelimb asymmetry test } & Days 4, 15, 20 \\
\hline \multicolumn{2}{|c|}{ Perfusion } & Day 28 \\
\hline
\end{tabular}

samples were separated with a microcentrifuge and stored frozen at $-80^{\circ} \mathrm{C}$. NAM was analyzed in serum using HPLC with UV detection at $254 \mathrm{~nm}$ on a Varian Pro Star 210 HPLC system using a modification of a previously developed assay used to measure NAD. ${ }^{34}$ Briefly, $60 \mu \mathrm{L}$ serum samples and the added internal standard, nicotinamide hypoxanthine dinucleotide sodium salt (de-amino NAD), were deproteinized with $1 \mathrm{M}$ perchloric acid, buffered with $1 \mathrm{M}$ potassium phosphate $\mathrm{pH}$ 7.5, and centrifuged. The supernatant was injected onto a Adsorbosil C-18, $5 \mathrm{um}$, $250 \times 4.6 \mathrm{~mm}$ column (Grace Davison Discovery Sciences, Deerfield, IL) and separated using a mobile phase gradient of $6 \%$ to $20 \%$ methanol over 10 mins in $0.2 \mathrm{M}$ ammonium phosphate at $\mathrm{pH}$ 5.25. The de-amino NAD eluted at 6.3 minutes and NAM eluted at 13.5 mins.

Histology. Twenty-eight days after CCI, animals were anesthetized with a lethal dose of urethane $(3.0 \mathrm{~g} / \mathrm{kg}$, i.p.). Once an absence of the pedal reflex response occurred, animals followed by $10 \%$ phosphate buffered formalin $(\mathrm{PBF})$. Once the brain was removed from the skull, they were post-fixed in PBF for 24 hours. Brains were then cryoprotected in a $30 \%$ sucrose solution for three days prior to frozen sectioning. Serial coronal sections (40 $\mu \mathrm{m}$ thick) were taken using a sliding microtome and a freezing stage. The sections were then collected into a cryopreservative solution, stored and then frozen.

Lesion analysis. The coronal sections were mounted on gelatin-subbed microscope slides and stained with cresyl violet to determine the extent of lesion cavity formation. An Olympus microscope (BX-51) with an Olympus 13.5 megapixel digital camera (DP-70) attached to it was used to capture images of four sections thoroughly traversing the lesion cavity (1.60, $0.70,-0.40,-1.60$ relative to bregma). ${ }^{35}$ The Calvalieri method was used to calculate the volume of the ipsilateral and contralateral cortex. ${ }^{36}$ The number of sections analyzed and their respective thickness $(40 \mu \mathrm{m})$ were multiplied by the mean area of the lesion cavity. The extent of cortical injury was calculated using the formula [100 - (ipsilateral cortex volume/contralateral cortex volume)*100]. This formula calculated the percent reduction in the ipsilateral cortex.

Data analysis. All behavioral testing and histological analyses were performed without knowledge of treatment parameters. 
Means and standard error of the means (SEM) were calculated for all data. The General Linear Model (GLM) ANOVA procedure with the repeated measures option was used to evaluate the effects of chronic NAM treatment across days. The groups included in the analysis were NAM-treated, vehicle-treated and sham. The between groups factor was treatment group assignment and the within groups factor was day of testing. The Huynh-Feldt correction was used to correct for repeated measures. Fischer's Least Significant Different (LSD) test was the pairwise comparison procedure used. One-way ANOVA's were calculated on the serum levels and anatomical data and were followed by appropriate post-hoc (LSD) analyses. Statistical significance was determined with a p value less than 0.05 .

\section{Acknowledgements}

We would like to thank Nicholas Kaufman for his help on this project. This research was funded by NIH grant NS045647 to M.R.H.

\section{References}

1. Langlois JA, Rutland-Brown W, Wald MM. The epidemiology and impact of traumatic brain injury: a brief overview. J Head Trauma Rehabil 2006; 21:10-7.

2. Thurman DJ, Alverson CA, Dunn KA, Guerrero J, Sniezek JE. Traumatic brain injury in the United States: a public health perspective. J Head Trauma Rehabil 1999; 14:602-15.

3. Gondusky JS, Reiter MP. Protecting military convoys in Iraq: an examination of battle injuries sustained by a mechanized battalion during Operation Iraqi Freedom II. Mil Med 2005; 170:546-9.

4. Narayan RK, Michel ME, Ansell B, Baethmann A, Biegon A, Bracken MB, et al. Clinical trials in head injury. J Neurotrauma 2002; 19:503-57.

5. Royo NC, Shimizu S, Schouten JW, Stover JF, McIntosh TK. Pharmacology of traumatic brain injury. Curr Opin Pharm 2003; 3:27-32.

6. Statler KD, Jenkins LW, Dixon CE, Clark RS, Marion DW, Kochanek PM. The simple model versus the super model: translating experimental traumatic brain injury research to the bedside. J Neurotrauma 2001; 18:1195206.

7. Sullivan PG, Keller JN, Mattson MP, Scheff SW. Traumatic brain injury alters synaptic homeostasis: implications for impaired mitochondrial and transport function. J Neurotrauma 1998; 15:789-98.

8. Verbois SL, Scheff SW, Pauly JR. Chronic nicotine treatment attenuates alpha7 nicotinic receptor deficits following traumatic brain injury. Neuropharmacology 2003; 44:224-33.

9. Cutler SM, Pettus EH, Hoffman SW, Stein DG. Tapered progesterone withdrawal enhances behavioral and molecular recovery after traumatic brain injury. Exp Neurol 2005; 195:423-9.

10. Hoane MR, Akstulewicz SL, Toppen J. Treatment with vitamin $\mathrm{B}_{3}$ improves functional recovery and reduces GFAP expression following traumatic brain injury in the rat. J Neurotrauma 2003; 20:1189-98.

11. Hoane MR, Gilbert DR, Holland MA, Pierce JL. Nicotinamide reduces acute cortical neuronal death and edema in the traumatically injured brain. Neurosci Lett 2006; 35-9.

12. Hoane MR, Kaplan SA, Ellis AL. The effects of nicotinamide on apoptosis and blood-brain barrier breakdown following traumatic brain injury. Brain Res 2006; 1125:185-93.
13. Hoane MR, Pierce JL, Holland MA, Anderson GD. Nicotinamide treatment induces behavioral recovery when administered up to 4 hours following cortical contusion injury in the rat. Neuroscience 2008; 154:861-8.

14. Hoane MR, Pierce JL, Kaufman NA, Beare JE. Variation in chronic nicotinamide treatment after traumatic brain injury can alter components of functional recovery independent of histological damage. Oxid Med Cell Longev 2008; 1:46-53.

15. Hoane MR, Tan AA, Pierce JL, Anderson GD, Smith DC. Nicotinamide treatment reduces behavioral impairments and provides cortical protection after fluid percussion injury in the rat. J Neurotrauma 2006; 23:1535-48.

16. Holland MA, Tan AA, Smith DC, Hoane MR Nicotinamide treatment provides acute neuroprotection and GFAP regulation following fluid percussion injury. J Neurotrauma 2008; 25:140-52.

17. Quigley A, Tan AA, Hoane MR. The effects of hypertonic saline and nicotinamide on sensorimotor and cognitive function following cortical contusion injury in the rat. Brain Res 2009; 1304:138-48

18. Clark RS, Vagni VA, Nathaniel PD, Jenkins LW, Dixon CE, Szabâo C. Local administration of the poly(ADPribose) polymerase inhibitor INO-1001 prevents NAD depletion and improves water maze performance after traumatic brain injury in mice. J Neurotrauma 2007; 24:1399-405.

19. Chong ZZ, Maiese K. Enhanced tolerance against early and late apoptotic oxidative stress in mammalian neurons through nicotinamidase and sirtuin mediated pathways. Curr Neurovasc Res 2008; 5:159-70.

20. Maiese K, Chong ZZ, Hou J, Shang YC. The vitamin nicotinamide: translating nutrition into clinical care. Molecules 2009; 14:3446-85

21. Sauve AA. $\mathrm{NAD}^{+}$and vitamin $\mathrm{B}_{3}$ : from metabolism to therapies. J Pharmacol Exp Ther 2008; 324:883-93.

22. Klaidman L, Morales M, Kem S, Yang J, Chang ML Adams JD Jr. Nicotinamide offers multiple protective mechanisms in stroke as a precursor for $\mathrm{NAD}^{+}$, as a PARP inhibitor and by partial restoration of mitochondrial function. Pharmacology 2003; 69:150-7.

23. Yang J, Klaidman LK, Adams JD. Medicinal chemistry of nicotinamide in the treatment of ischemia and reperfusion. Mini Rev Med Chem 2002; 2:125-34.
24. Ayoub IA, Lee EJ, Ogilvy C, Flint Beal M, Maynard KI. Nicotinamide reduces infarction up to two hours after the onset of permanent focal cerebral ischemia in wistar rats. Neurosci Lett 1999; 259:21-4.

25. Ayoub IA, Maynard KI. Therapeutic window for nicotinamide following transient focal cerebral ischemia. Neuroreport 2002; 11:213-6.

26. Yang J, Klaidman LK, Chang ML, Kem S, Sugawara $T$, Chan P, et al. Nicotinamide therapy protects against both necrosis and apoptosis in a stroke model. Pharmacol Biochem Behav 2002; 73:901-10.

27. Maiese K, Chong ZZ. Nicotinamide: necessary nutrient emerges as a novel cytoprotectant for the brain. Trends Pharmacol Sci 2003; 24:228-32.

28. Spector R. Niacin and nicotinamide transport in the central nervous system. J Neurochem 1979; 33:895904

29. Maynard KI, Ayoub IA, Shen C. Delayed multidose treatment with nicotinamide extends the degree and duration of neuroprotection by reducing infarction and improving behavioral scores up to two weeks following transient focal cerebral ischemia in wistar rats. Ann $\mathrm{N}$ Y Acad Sci 2001; 939:416-24.

30. Li F, Chong ZZ, Maiese K. Cell Life versus cell longevity: the mysteries surrounding the $\mathrm{NAD}^{+}$precursor nicotinamide. Curr Med Chem 2006; 13:883-95.

31. Ungerstedt JS, Blomback $M$, Soderstrom T. Nicotinamide is a potent inhibitor of proinflammatory cytokines. Clin Exp Immunol 2003; 131:48-52.

32. Hoane MR, Pierce JL, Holland MA, Birky ND, Dang T, Vitek MP, et al. The novel apolipoprotein E-based peptide COG1410 improves sensorimotor performance and reduces injury magnitude following cortical contusion injury. J Neurotrauma 2007; 24:1108-18.

33. Schallert T, Woodlee MT. Orienting and Placing. In: Whishaw IQ, Kolb B, eds. The Behavior of the Laboratory Rat. New York: Oxford University Press 2005; 129-40.

34. Kalhorn TF, Thummel KE, Nelson SD, Slattery JT Analysis of oxidized and reduced pyridine dinucleotides in rat liver by high-performance liquid chromatography. Ann Biochem 1985; 151:343-7.

35. Paxinos G, Watson C. The rat brain in stereotaxic coordinates. New York: Elsevier 2005.

36. Coggeshall RE. A consideration of neural counting methods. TINS 1992; 15:9-13. 


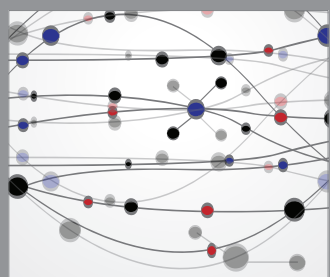

The Scientific World Journal
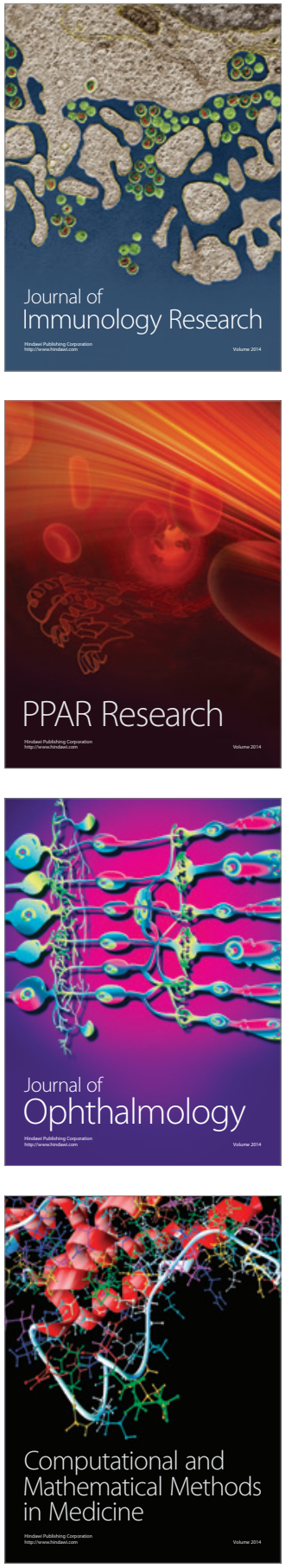

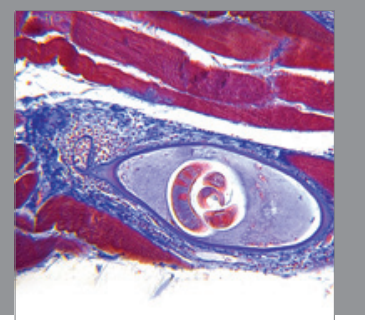

Gastroenterology

Research and Practice
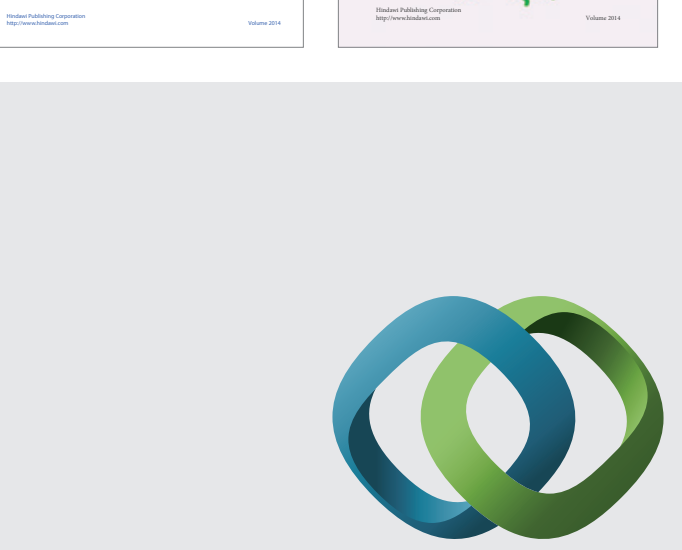

\section{Hindawi}

Submit your manuscripts at

http://www.hindawi.com
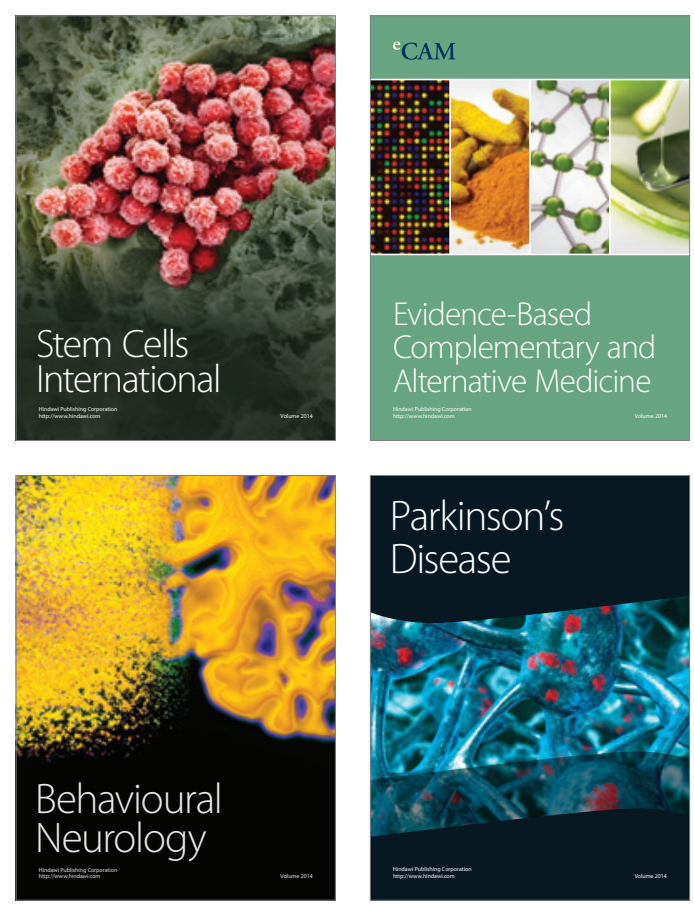

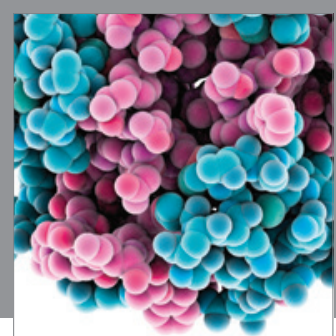

Journal of
Diabetes Research

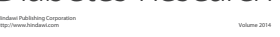

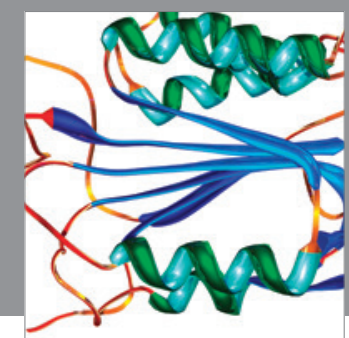

Disease Markers
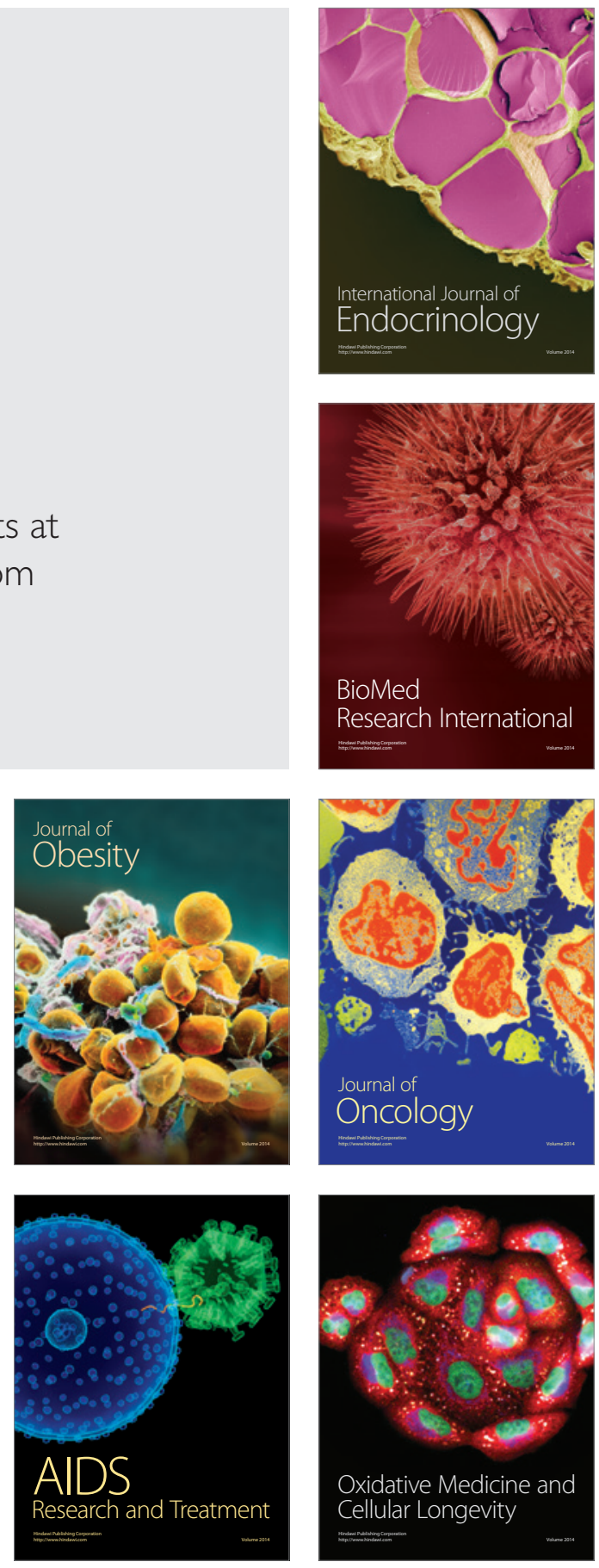